\title{
The Relationship Between Omega-3, Omega- 6 and Total Polyunsaturated Fat and Musculoskeletal Health and Functional Status in Adults: A Systematic Review and Meta-analysis of RCTs
}

\author{
Asmaa Abdelhamid ${ }^{1} \cdot$ Lee Hooper ${ }^{1} \cdot$ Ruksana Sivakaran $^{1} \cdot$ Richard P. G. Hayhoe $^{1}$ - Ailsa Welch ${ }^{1}$ on behalf of the \\ PUFAH Group
}

Received: 5 June 2019 / Accepted: 5 July 2019 / Published online: 25 July 2019

(c) The Author(s) 2019

\begin{abstract}
We conducted a systematic review and meta-analysis to assess the effects of increasing dietary omega-3, omega-6 and mixed polyunsaturated fatty acids (PUFA) on musculoskeletal health, functional status, sarcopenia and risk of fractures. We searched Medline, Embase, The Cochrane library, ClinicalTrials.gov and the WHO International Clinical Trials Registry Platform (ICTRP) databases for Randomised Controlled Trials (RCTs) of adults evaluating the effects of higher versus lower oral omega-3, omega- 6 or mixed PUFA for $\geq 6$ months on musculoskeletal and functional outcomes. We included 28 RCTs (7288 participants, 31 comparisons), 23 reported effects of omega-3, one of omega- 6 and four of mixed total PUFA. Participants and doses were heterogeneous. Six omega-3 trials were judged at low summary risk of bias. We found low-quality evidence that increasing omega-3 increased lumbar spine BMD by $2.6 \%\left(0.03 \mathrm{~g} / \mathrm{cm}^{2}, 95 \% \mathrm{CI}-0.02\right.$ to $0.07,463$ participants $)$. There was also the suggestion of an increase in femoral neck BMD (of 4.1\%), but the evidence was of very low quality. There may be little or no effect of omega-3 on functional outcomes and bone mass; effects on other outcomes were unclear. Only one study reported on effects of omega-6 with very limited data. Increasing total PUFA had little or no effect on BMD or indices of fatfree (skeletal) muscle mass (low-quality evidence); no data were available on fractures, BMD or functional status and data on bone turnover markers were limited. Trials assessing effects of increasing omega-3, omega-6 and total PUFA on functional status, bone and skeletal muscle strength are limited with data lacking or of low quality. Whilst there is an indication that omega-3 may improve BMD, high-quality RCTs are needed to confirm this and effects on other musculoskeletal outcomes.
\end{abstract}

Keywords Fatty acids omega-3 $\cdot$ Musculoskeletal physiological phenomena $\cdot$ Bone density $\cdot$ Muscle strength $\cdot$ Fatty acids omega-6 $\cdot$ Fatty acids unsaturated $\cdot$ Meta-analysis $\cdot$ Randomised controlled trial $\cdot$ Aged $\cdot$ Alpha-linolenic acid · Docosahexaenoic acids $\cdot$ Eicosapentaenoic acid

Systematic review registration - PROSPERO 2017 CRD42017079211.

The members of the PUFAH group are listed in Acknowledgement section.

Electronic supplementary material The online version of this article (https://doi.org/10.1007/s00223-019-00584-3) contains supplementary material, which is available to authorized users.

Asmaa Abdelhamid

Asmaa.abdelhamid@uea.ac.uk

$\triangle$ Lee Hooper

1.hooper@uea.ac.uk

Extended author information available on the last page of the article

\section{Introduction}

Decline in musculoskeletal health presents a significant risk to functional ability for older individuals, with concomitant reduction in quality of life, greater demand on health and social care services and higher risk of mortality. Sarcopenia (loss of skeletal muscle mass and strength), loss of bone mass and structural integrity (osteopenia or osteoporosis) are common in later life and are partially inter-dependent [1, 2], with each contributing to increased frailty, physical disability, risk of falls and fractures [3, 4]. Loss of bone mass starts in adults around 30 to 40 years of age and women in the post-menopausal decade experience a particularly high rate of decline. Men also experience progressive, albeit smaller, loss of bone mass [5]. Loss of skeletal muscle mass and 
function and the development of sarcopenia follow similar trajectories to bone [6]. Together sarcopenia and fractures have high prevalence and economic burden $[7,8]$. This is predicted to increase so that by $2045,13 \%$ to $22 \%$ of those aged $>65$ years in Europe will be sarcopenic [9], and 20\% of women and $5 \%$ of men aged $>50$ years will have osteoporosis. Currently, approximately half of women and a fifth of men over the age of 60 years will experience an osteoporotic fracture [7]. As the number of individuals aged $\geq 60$ is expected to double worldwide by 2050 [10] development and implementation of effective strategies to reduce the global burden of musculoskeletal decline is critical to avoid unsustainable demands on future health and social care systems. Dietary intervention to reduce or delay musculoskeletal decline may represent a relatively easily achievable component of such strategies, and thus it is of particular public health importance to further our understanding of how nutritional factors contribute to musculoskeletal health during ageing.

Dietary fat is important to normal muscle structure and function. Fatty acids act as a key substrate for ATP production and thus provide a major energy source during aerobic exercise [11], as well as being key structural components of the sarcolemma (muscle cell membrane). Several mechanisms have been proposed for omega- 3 and omega- 6 polyunsaturated fatty acids (PUFA) in musculoskeletal health [12-14]. These include PUFA-induced maintenance of antioxidant-oxidant balance, preventing the oxidative stress that can lead to skeletal muscle atrophy; omega-6-induced activation of transcription factor peroxisome proliferator activator receptor gamma (PPAR $\gamma$ ), which inhibits osteoblast growth, negatively affecting bone remodelling [15]; omega-3 effects on increasing calcium absorption, by modulating calcium-ATPase when levels are low [16]; PUFAs promoting osteoblastic differentiation through increased production of IGF-1 and parathyroid hormone and omega-3 FAs causing downregulation of chemicals involved in osteoclastic growth $[13,17]$.

Overall, omega-3 is suggested to exhibit protective effects on bone and muscle whilst omega- 6 is thought to have proinflammatory effects with detrimental consequences to musculoskeletal health. Thus, a higher omega-3: omega-6 ratio is proposed as beneficial. This is supported by studies showing populations with high omega-3: omega-6 ratios, such as the Japanese or Inuit, have lower rates of osteoporosis than populations with lower omega-3: omega-6 dietary ratios [18].

Previous systematic reviews evaluating the effects of omega-3, omega- 6 or total PUFA on skeletal muscle and bone health, sarcopenia or fracture risk have been limited to specific PUFAs (usually omega-3) [19-22], specific population subgroups (e.g. post-menopausal women) [19] or specific outcomes (e.g. bone markers or osteoporosis) [23] and most did not meta-analyse. Effects of total PUFA have not been investigated recently [24]. There is thus a need for a comprehensive review of omega-3, omega- 6 and mixed PUFAs on bone and skeletal muscle health or functional status, in order to inform public health nutritional policy for musculoskeletal health in older individuals. We therefore undertook this systematic review to assess effects of increasing dietary omega-3, omega- 6 or mixed total PUFA on key musculoskeletal outcomes and functional status in adults aged 40 years or older.

\section{Methods}

We conducted the review following methods recommended by The Cochrane Handbook and reported the process and results in accordance with the preferred reporting items for systematic reviews and meta-analyses (PRISMA) statement $[25,26]$. This review was part of a set of reviews evaluating the effects of PUFAs on various health outcomes [27-34]; detailed methods are reported elsewhere [35]. The methods reported below are those specific to this review.

\section{Selection Criteria}

We included randomised controlled trials (RCTs) that compared higher versus lower omega-3, omega- 6 or mixed PUFA (i.e. providing both omega-3 and omega-6), over a period of at least 24 weeks. We included studies reported as full text, trials registry entries and conference abstracts. Participants included healthy adults and those at risk of or diagnosed with sarcopenia and/or osteopenia, aged $\geq 40$ years (where $\geq 90 \%$ of participants were aged $\geq 40$ years or where data could be separated out for those aged $\geq 40$ ). Studies of pregnant women or acutely ill patients were excluded. Participants with non-acute co-morbidities were included except where the co-morbidity was likely to affect fatty acid absorption or musculoskeletal outcomes (e.g. coeliac disease, cystic fibrosis, systemic lupus erythematosus, rheumatoid arthritis, ulcerative colitis and Crohn's Disease).

Interventions had to aim to increase or decrease omega3 , omega- 6 and/or total PUFA intakes, or achieve $\geq 10 \%$ increase or decrease from baseline. Interventions could include dietary supplementation (oils, capsules, enriched foods or naturally rich food sources given by mouth), or provided diet or dietary advice. Multifactorial interventions (with exercise, smoking cessation, medications or other dietary aims) were excluded unless the effect of change in PUFA could be separated out from other interventions. The control group had to have lower PUFA intake (including usual diet; no advice; no supplementation; placebo or an intervention aiming at lowering PUFA intake). Primary outcomes included: 
- For bone fracture incidence, any measure of BMD, e.g. bone mineral density (BMD), bone mineral content (BMC) or total bone mass.

- For muscle sarcopenia or dynapenia (age-associated loss of muscle strength) incidence, skeletal muscle mass.

- For functional status mobility scores or other validated functional status measures, e.g. Barthel Index or Activities of Daily Living (ADL).

Secondary outcomes included direct measures of muscle strength or physical performance (e.g. grip strength, gait speed), fracture risk score, osteoporosis or osteopenia incidence and bone turnover markers. A study was eligible for inclusion if it assessed any primary or secondary outcome.

\section{Search Methods for Identification of Studies}

We identified studies using complex and extensive search strategies and duplicate assessment as described elsewhere [35], creating a database of trials that randomised participants to increased omega-3, omega- 6 or total PUFA compared to lower omega-3, omega- 6 or total PUFA and assessed effects over at least 24 weeks. From the database, studies were chosen for this review that had assessed any primary or secondary outcome (even when not fully reported). Reference lists of all included primary studies and relevant systematic reviews were hand-searched for additional references.

\section{Data Collection and Assessment of Risk of Bias}

Two reviewers independently assessed inclusion, extracted data (characteristics, methods and results data) and assessed risk of bias. Risk of bias was assessed using the Cochrane Risk of Bias tool [36]. A trial was considered to be at low summary risk of bias where randomisation, allocation concealment, blinding of participants and personnel and blinding of outcome assessors were all judged adequate [35]. We tried to contact authors where inclusion could not be ascertained, where outcomes were measured but not reported or not reported in a usable format.

\section{Data Synthesis}

Our primary analyses assessed effects of total PUFA, omega-6, omega- 3 on our primary outcomes. Treatment versus control differences in outcomes were combined across studies where appropriate using relative risks (RR) or mean differences (MD) in random-effects meta-analysis (using Review Manager Version 5.3; The Nordic Cochrane Centre, The Cochrane Collaboration, Copenhagen, Denmark). The random-effects model was used since dietary interventions are heterogeneous. Change from baseline was preferred; however, we used end data where change data were not reported or reported with no variance. Where different scales measured the same outcome we ensured all scales worked in the same direction (inverting where necessary), before combining data using standardised mean differences (SMD). For SMDs, 0.2 represented little or no effect, 0.5 a moderate effect and 0.8 a large effect. Where a representative study at low summary risk of bias was included in the meta-analysis, we translated the pooled SMD back into the scale used in that trial to help understand effect sizes.

Subgroup analyses were planned to explore the effects of the long-chain omega 3 (LCn-3) EPA (eicosapentaenoic acid) and DHA (docosahexaenoic acid), their precursor alpha linoleic acid (ALA), omega-6 and mixed PUFA intake on primary outcomes where at least ten RCTs reported a single comparison. Planned subgroup analyses included gender, baseline risk of osteoporosis/skeletal muscle loss (general population; those at higher risk, e.g. post-menopausal women, early menopause, $65+$, family history; those with osteopenia/sarcopenia; those with diagnosed osteoporosis), intervention type, trial duration, baseline LCn-3, ALA, omega-6 or total PUFA intake [35]. Sensitivity analyses were carried out to assess the effects of methodological rigour (including only studies with a low summary risk of bias), study size and fixed effects meta-analysis. Funnel plots were planned to explore potential reporting biases for the primary outcomes where we included $\geq 10$ studies in single meta-analyses.

The GRADE (Grading of Recommendations, Assessment, Development and Evaluations) framework was used to assess strength of evidence across studies for primary outcomes. Outcome data were interpreted as usual for this set of reviews [35]. RR $<0.92$ or $>1.08$ was considered an effect, whilst change from baseline of $\geq 5 \%$ was required for continuous measures except for cumulative measures such as BMD and adiposity (where a change of $\geq 2 \%$ was required). This $2 \%$ change compares with changes of $0.4 \%$ annual decrease in BMD in older adults and the 5\% decrease with $3 \%$ annual decreases in grip strength in older adults [37].

\section{Results}

Study flow from 37,810 titles and abstracts generated by electronic searches through to our database of 363 RCTs of at least 6 months duration comparing higher with lower omega-3, omega- 6 and/or total PUFA intake has been detailed previously [35]. Of the 363 RCTs in the database 28 RCTs (7288 participants, 31 comparisons) met our inclusion criteria, of which 25 RCTs (27 comparisons) contributed to meta-analyses (see Fig. 1 for the flow diagram). Characteristics of these 28 included studies are summarised in Table 1, full details are in the database [35]. Fourteen included RCTs 
Fig. 1 Study flow diagram

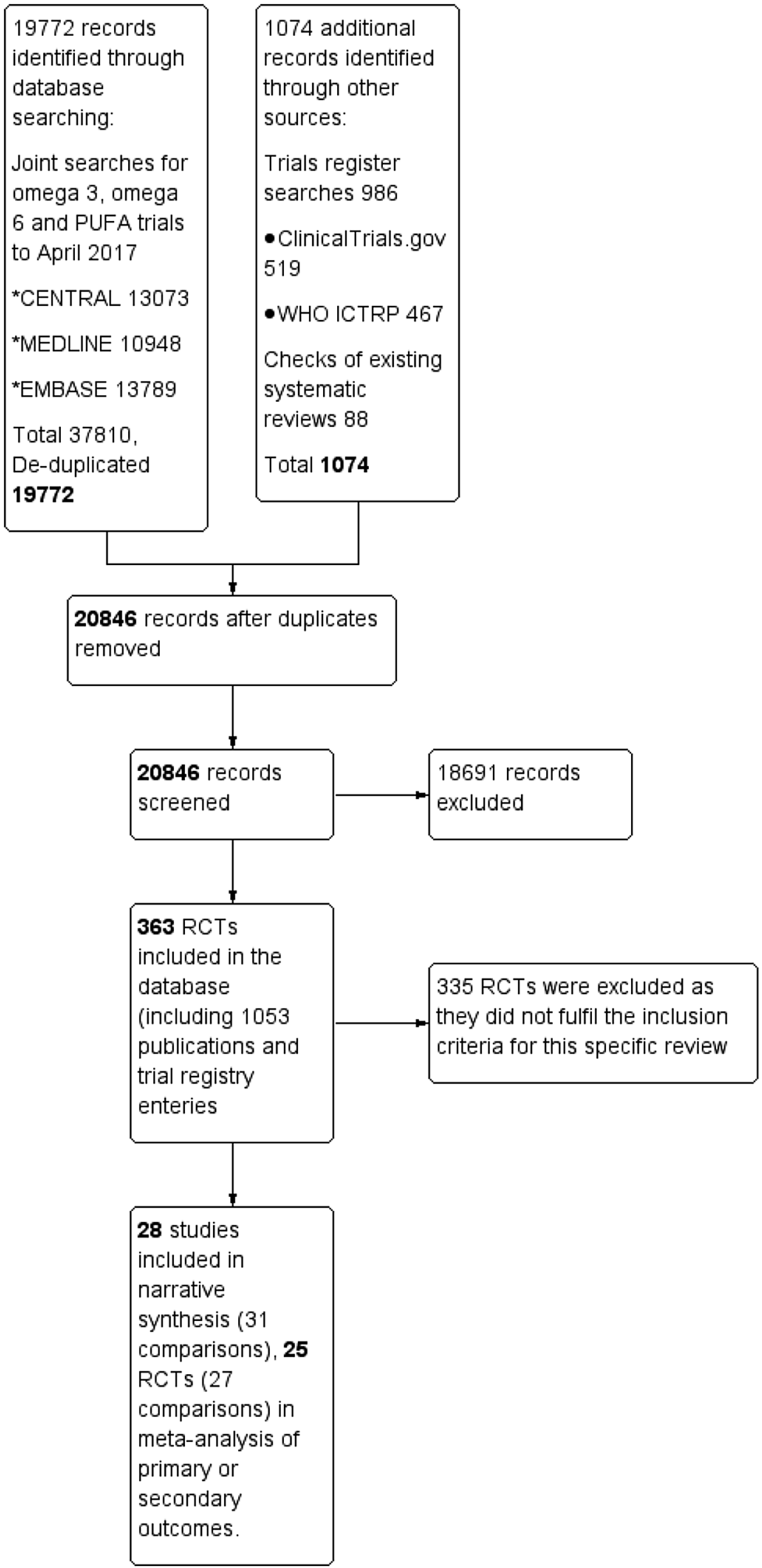




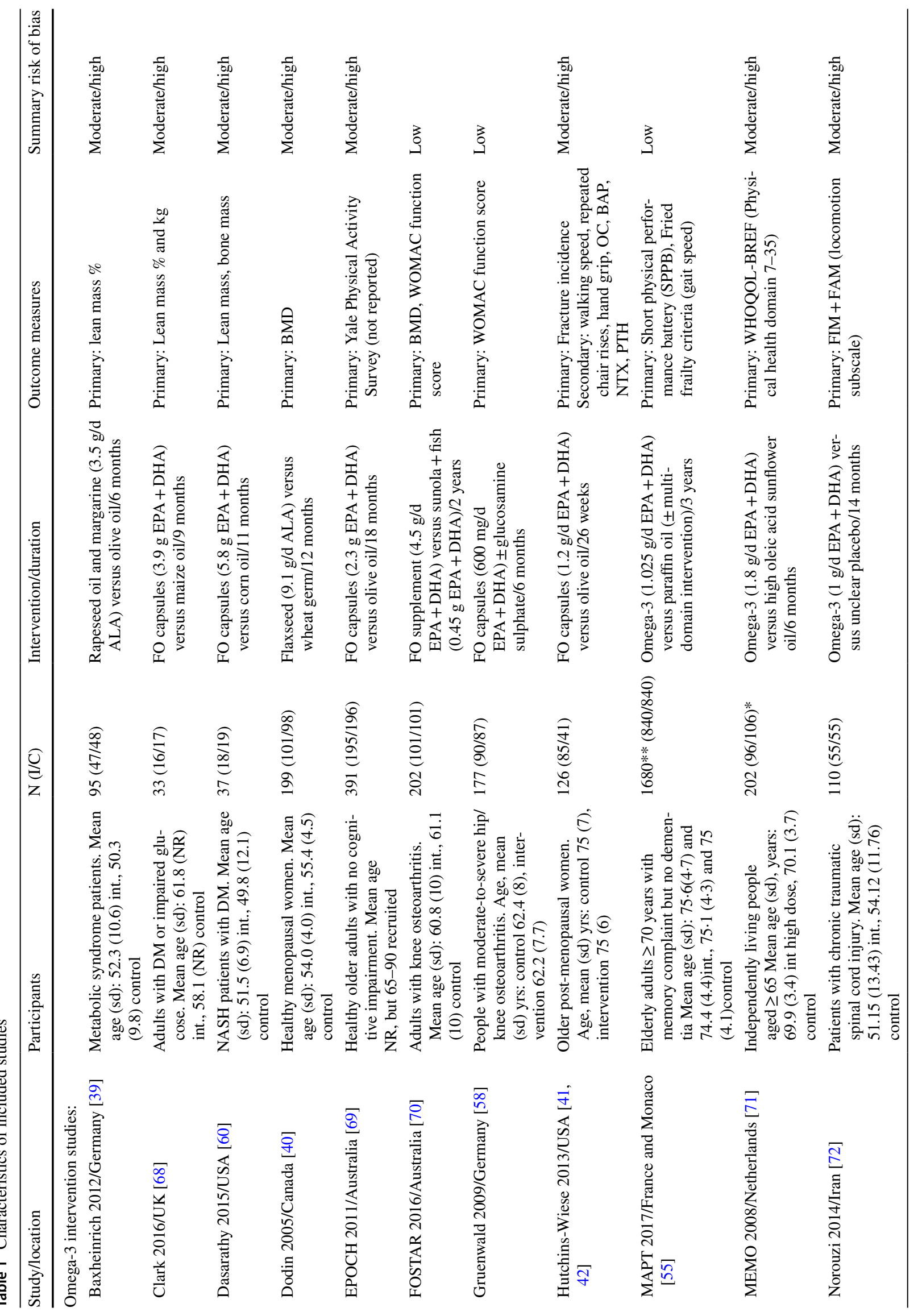




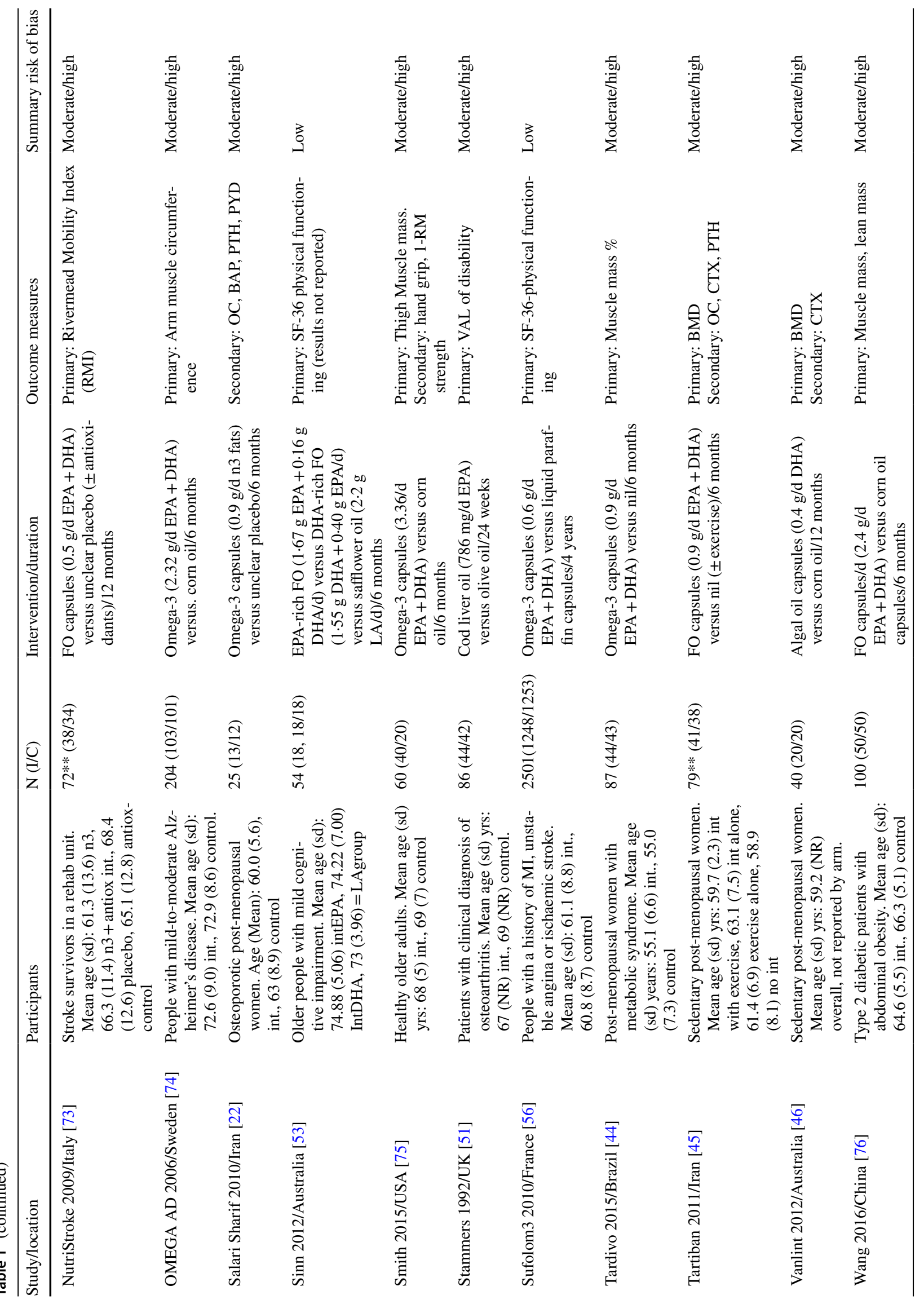




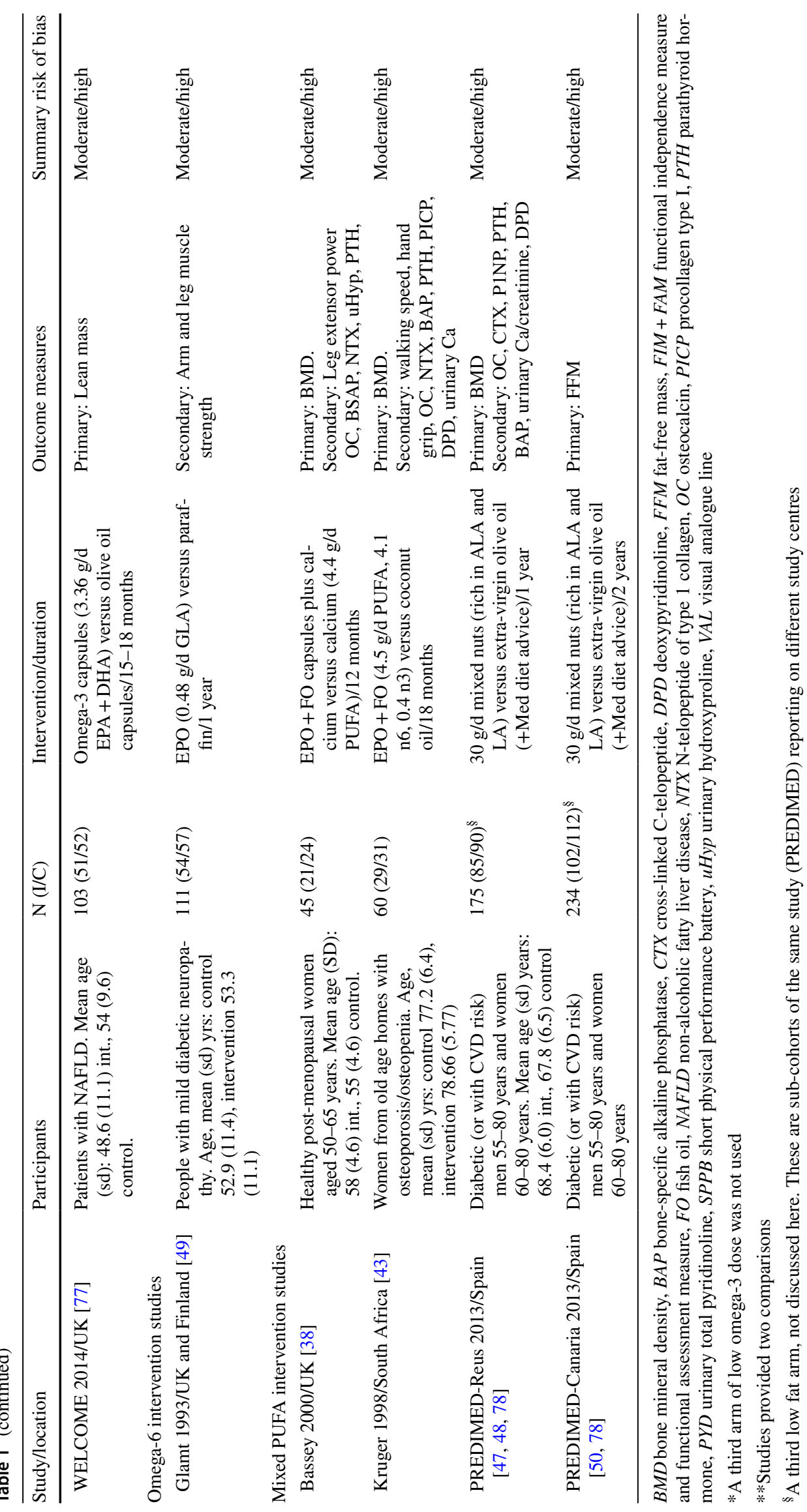


were conducted in Europe, four in North America, four each in Asia and Australia and one each for South America and Africa. Eight RCTs included only post-menopausal women [22, 38-46], two of which were of osteopenic or osteoporotic women [22, 43]. Sample sizes ranged from 25 to 2501 (mean 252, median 100 participants), and intervention duration ranged from 24 weeks to 4 years (mean 12.9 months).

Twenty-three studies were omega- 3 interventions, of which 21 provided LCn-3 (EPA and/or DHA) and two provided ALA [39, 40]. Effects of increasing mixed total PUFA was evaluated by three studies [38, 43, 47, 48], whilst omega-6 was assessed in by one [49]. The intervention was supplementary capsules in 23 studies with the remaining five providing supplementary oils, nuts or seeds [39, 40, 47, $48,50,51]$. No studies provided dietary advice only. Doses ranged from 0.4 to $5.8 \mathrm{~g} / \mathrm{d} \mathrm{LCn}-3$ and 3.5 to $9.1 \mathrm{~g} / \mathrm{d}$ ALA. The omega- 6 study provided $0.48 \mathrm{~g} / \mathrm{d}$ GLA and two mixed PUFA studies provided $\sim 4.5 \mathrm{~g} / \mathrm{d}$ (PUFA dose was unclear in one study).

Data from three studies could not be used in the metaanalysis. EPOCH and Sinn did not report numerical data for functional outcomes [52, 53], whilst OmegAD only provided arm muscle circumference as medians and interquartile range [54]. Six included RCTs, assessing effects of omega-3 [46, 53, 55-59], were judged to be at low summary risk of bias (Fig. 2).

\section{Effects of Higher Omega-3}

GRADE assessment of quality of evidence is shown in Table 2, and the meta-analysis results in Additional Table 1. No studies reported data on BMC, sarcopenia, dynapenia or myopenia incidence.

\section{Fractures}

The effect of increasing omega-3 fats on fracture incidence is unclear as the evidence is of very low quality (downgraded once for risk of bias, twice for imprecision). One RCT [42] reported the incidence of one fracture (RR $0.16,95 \%$ CI 0.01 to $3.91,126$ participants).

\section{Bone Density}

Increasing omega-3 intake may have little or no effect on total bone mass, with data available from one small study [60]. Total bone mass increased $<2 \%$ from baseline $(0.2 \mathrm{~kg}$, $95 \% \mathrm{CI}-2.8$ to 3.2 ). Evidence was of low quality, downgraded once each for risk of bias (the study was not at low summary risk of bias) and imprecision (as the 95\% CI included both important benefits and harms).

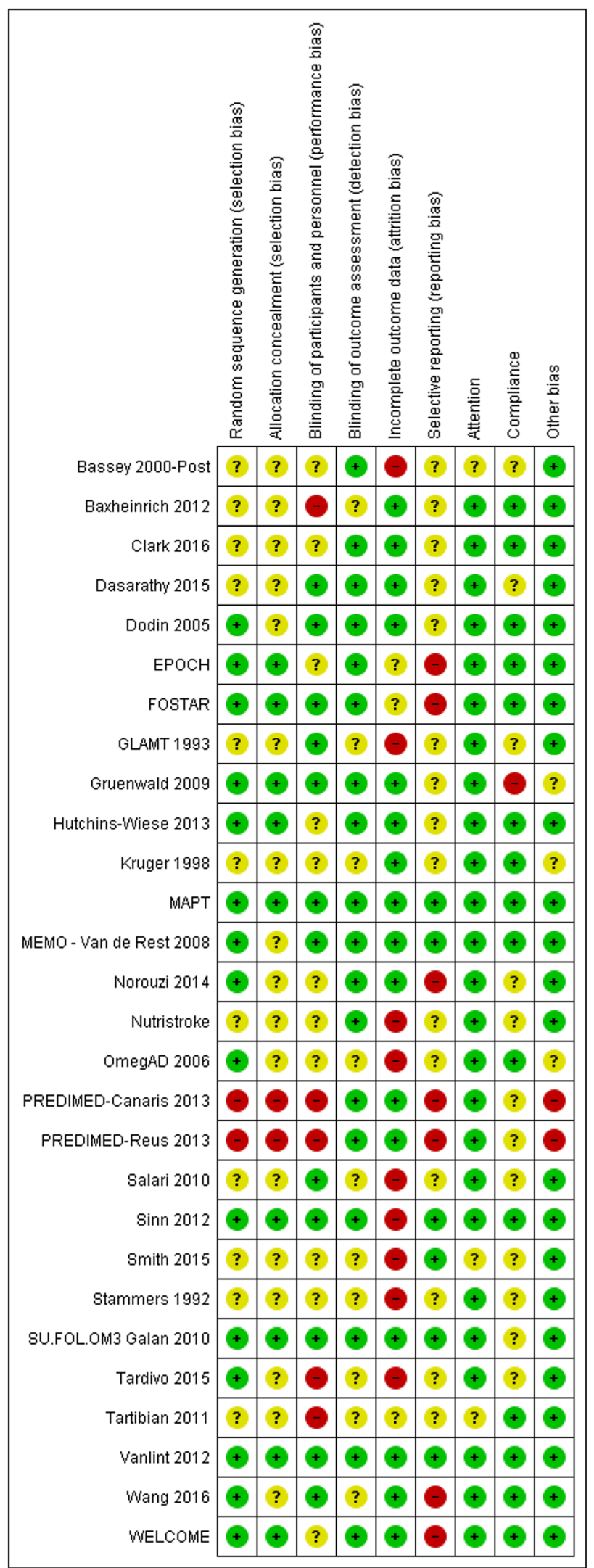

Fig. 2 Risk of bias summary for each included study 


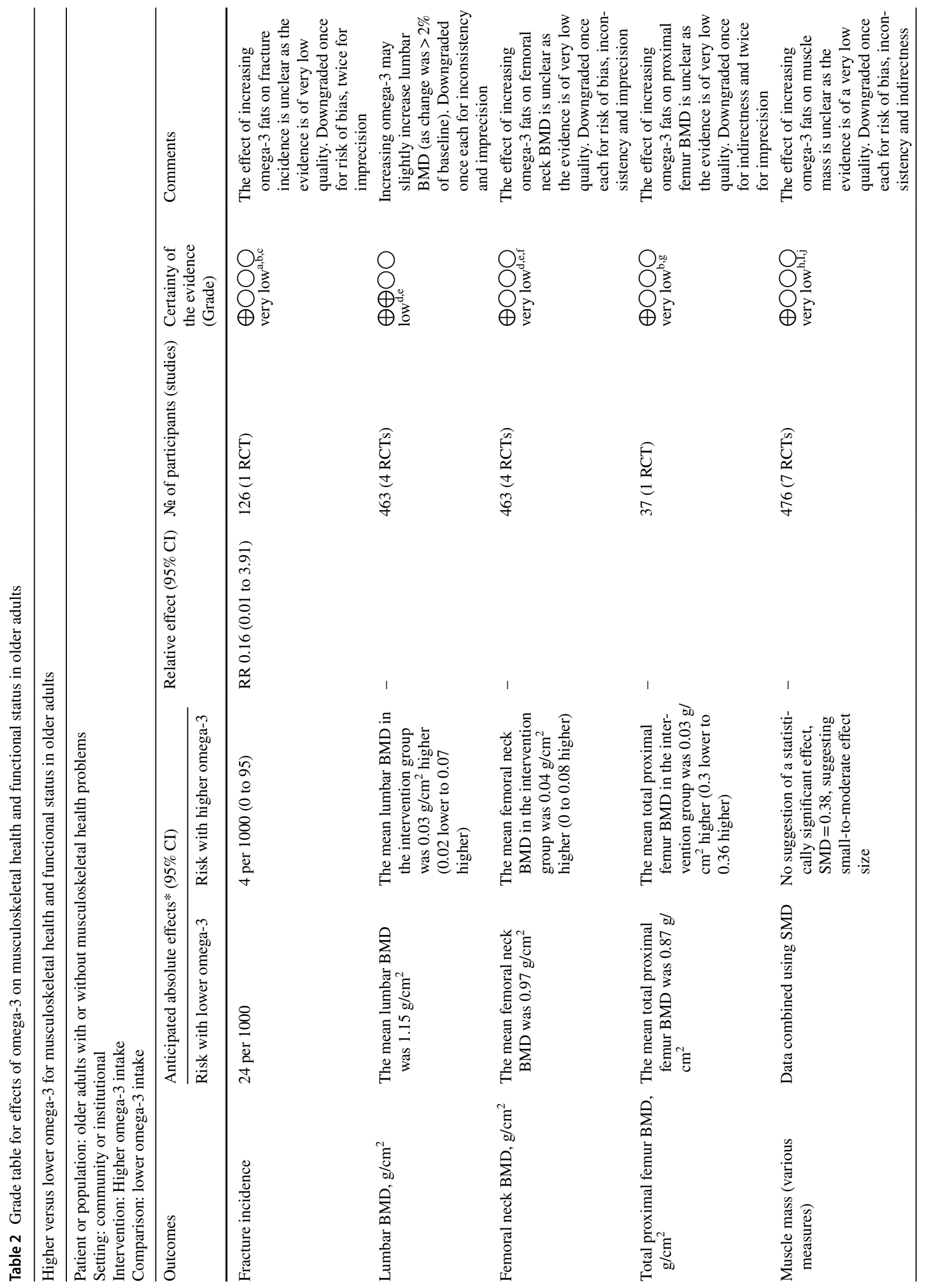




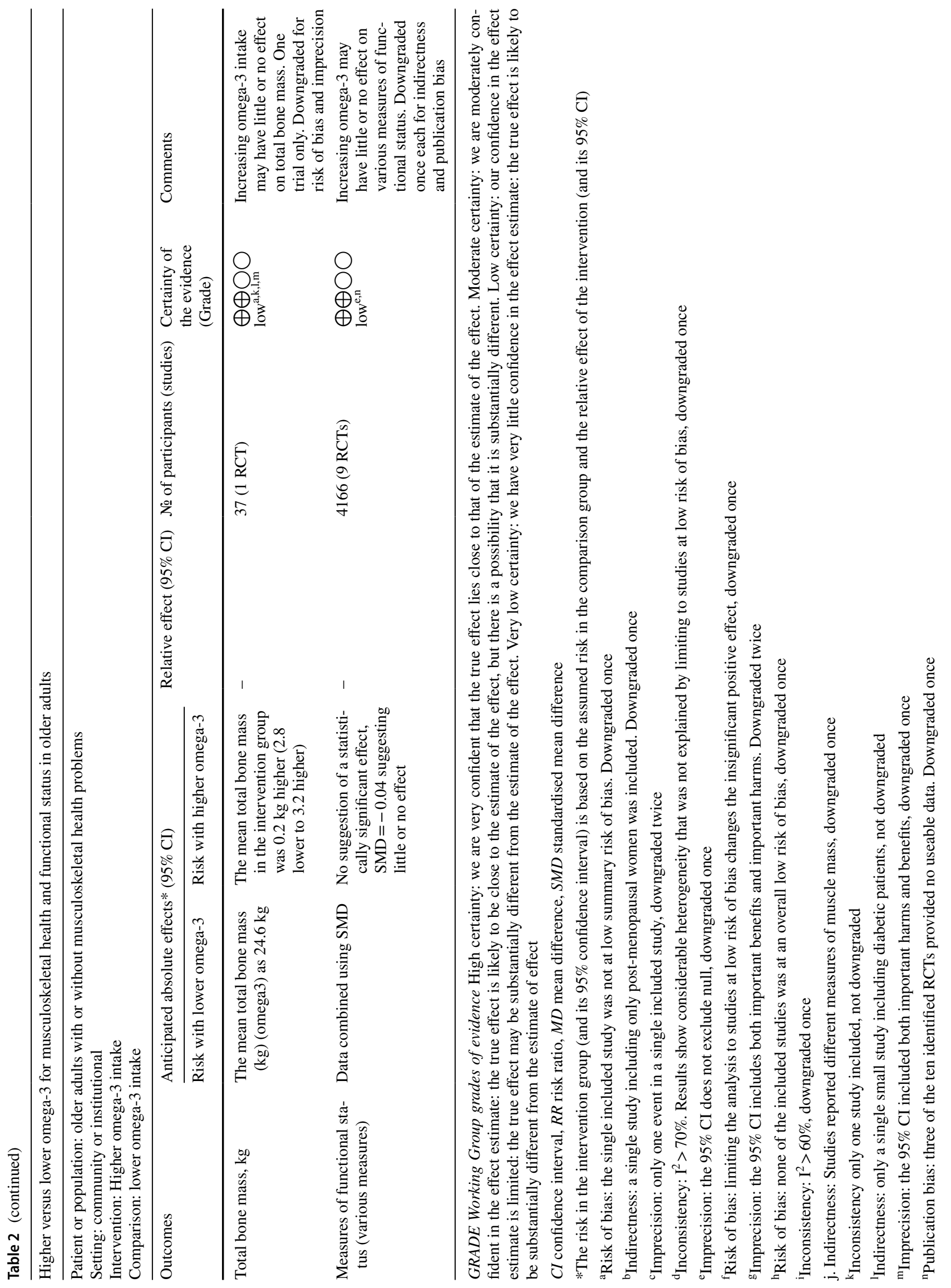




\section{Bone Mineral Density (BMD)}

Effects of increasing omega-3 fats on proximal femur BMD are unclear as the evidence is of very low-quality downgraded once for indirectness, twice for imprecision. Increasing omega- 3 may slightly increase lumbar spine BMD by $2.6 \%$ (MD $0.03 \mathrm{~g} / \mathrm{cm}^{2}, 95 \% \mathrm{CI}-0.02$ to $0.07,463$ participants, low-quality evidence, downgraded once each for inconsistency and imprecision) and femoral neck BMD by $4.1 \%$ (MD $0.04 \mathrm{~g} / \mathrm{cm}^{2}, 95 \%$ CI 0.0 to $0.08,463$ participants, very low-quality evidence, downgraded once each for risk of bias, inconsistency and imprecision). Four omega-3 studies (5 comparisons, of which 4 were in post-menopausal women) reported effects on BMD (Fig. 3). There was little or no effect of omega-3 on BMD at any site when sensitivity analyses were limited to RCTs at low summary risk of bias (Additional Table 1). We ran a post hoc sensitivity analysis limiting to the three studies of at least 1 year (as BMD changes slowly). This led to losing the effect on lumbar spine BMD (MD $0.00 \mathrm{~g} / \mathrm{cm} 2,95 \%$ CI -0.04 to 0.04 , 384 participants) and decreasing the effect shown on femoral neck BMD (MD $0.01 \mathrm{~g} / \mathrm{cm}^{2}, 95 \%$ CI 0.0 to $0.02,384$ participants).
As the same studies reported BMD at several sites, SMD was not used to combine results.

\section{Skeletal Muscle Mass}

The effect of increasing omega- 3 fats on skeletal muscle mass is unclear as the evidence is of a very low quality. Indices of skeletal muscle mass were reported in nine studies as percentage, percentage change or in kilograms, so we used SMD to combine measures, suggesting a small statistically significant improvement in indices muscle mass with higher omega- 3 and a small-to-moderate effect size (Fig. 4). The evidence was undermined by high heterogeneity (SMD $0.38,95 \%$ CI 0.05 to $0.70,7$ studies, 476 participants, $\mathrm{I}^{2} 67 \%$ ), risk of bias (as no included studies were at low summary risk of bias) and indirectness (measures varied between studies). We identified one study with missing data [54] which reported slightly higher median arm muscle circumference in the omega- 3 arm compared to control (change of $-0.1 \mathrm{~cm}$ in males and $+0.1 \mathrm{~cm}$ in females, compared to $-0.3 \mathrm{~cm}$ in placebo for males and females combined).

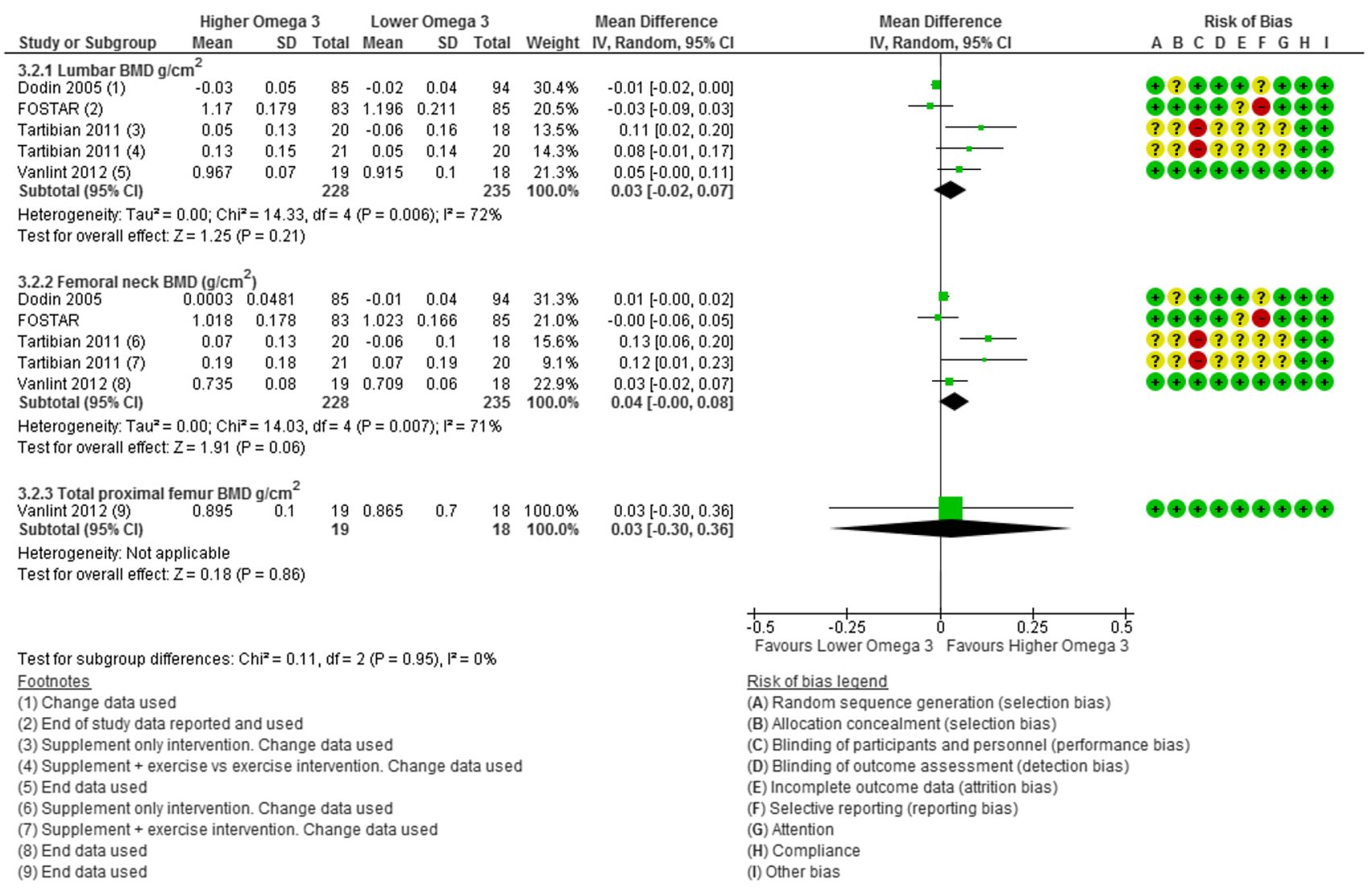

Fig. 3 Effect of high versus low omega-3 on BMD at different sites 


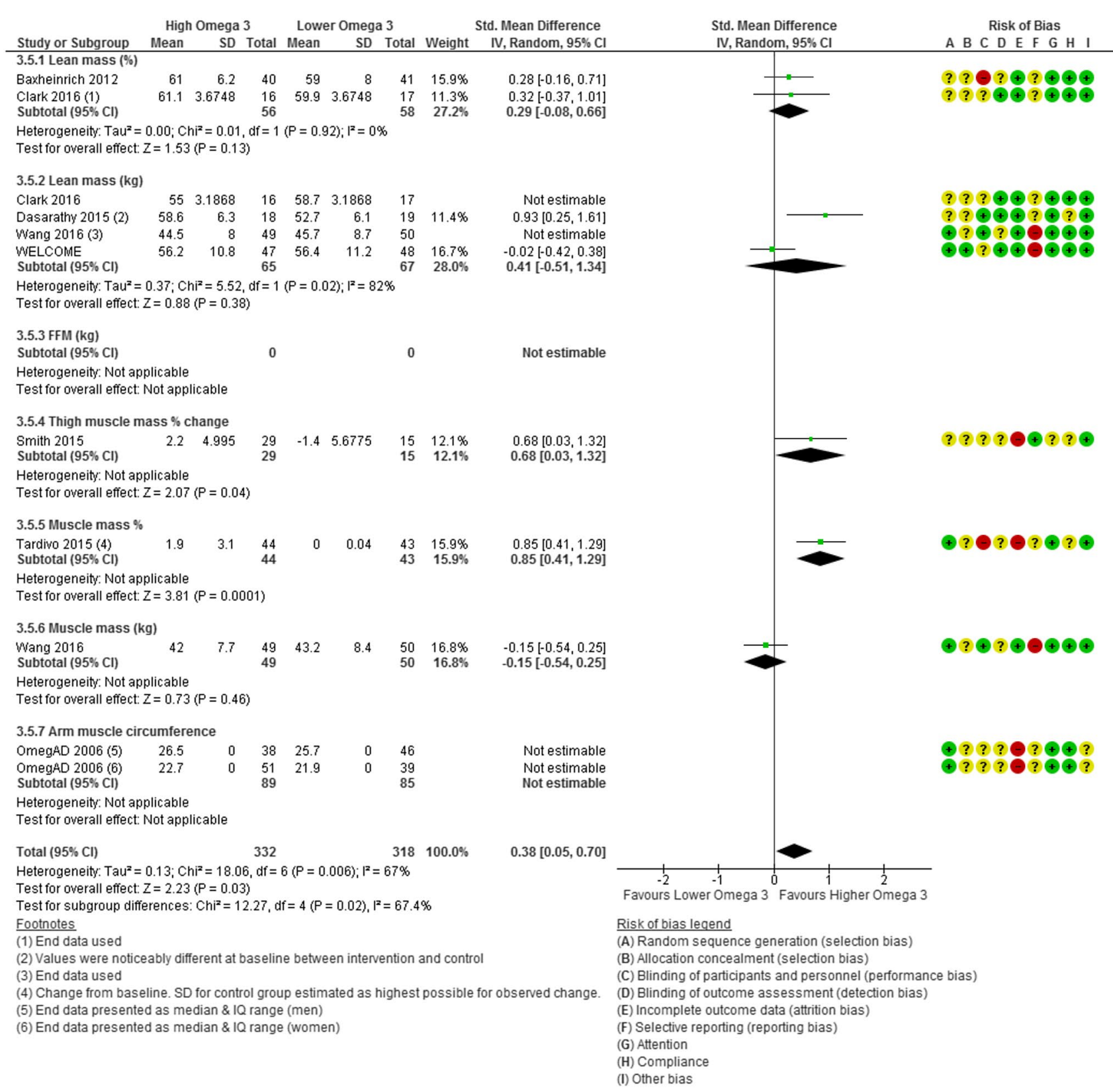

Fig. 4 Effect of high versus low omega-3 on various measures of skeletal muscle mass in different sites

\section{Functional Status}

Increasing omega-3 may have little or no effect on functional status (low-quality evidence, downgraded once each for indirectness and publication bias). Ten RCTs measured functional status using various scales, of which eight presented numerical results of which one presented means without variance. We used SMD to combine the remaining seven RCTs [9 comparisons, SMD-0.04, 95\% CI $-0.11,0.02, \mathrm{I}^{2}$ 0\% (Fig. 5)]. This SMD equates to little or no effect, and using MAPT Short Physical Performance Battery Score to re-express SMD suggests MD 0.08 equates to $0.7 \%$ increase from baseline on the SPPB scale. The three studies without usable data suggested non-statistically significant effects [53, 58 ] or provided no data [52].

\section{Secondary Outcomes}

\section{Measures of Physical Performance/Strength}

Two studies reported measures of handgrip strength (\% change or $\mathrm{kg}$ ), neither was at low summary risk of bias. Combining these using SMD did not suggest a statistically significant effect of increased omega-3. Other measures 


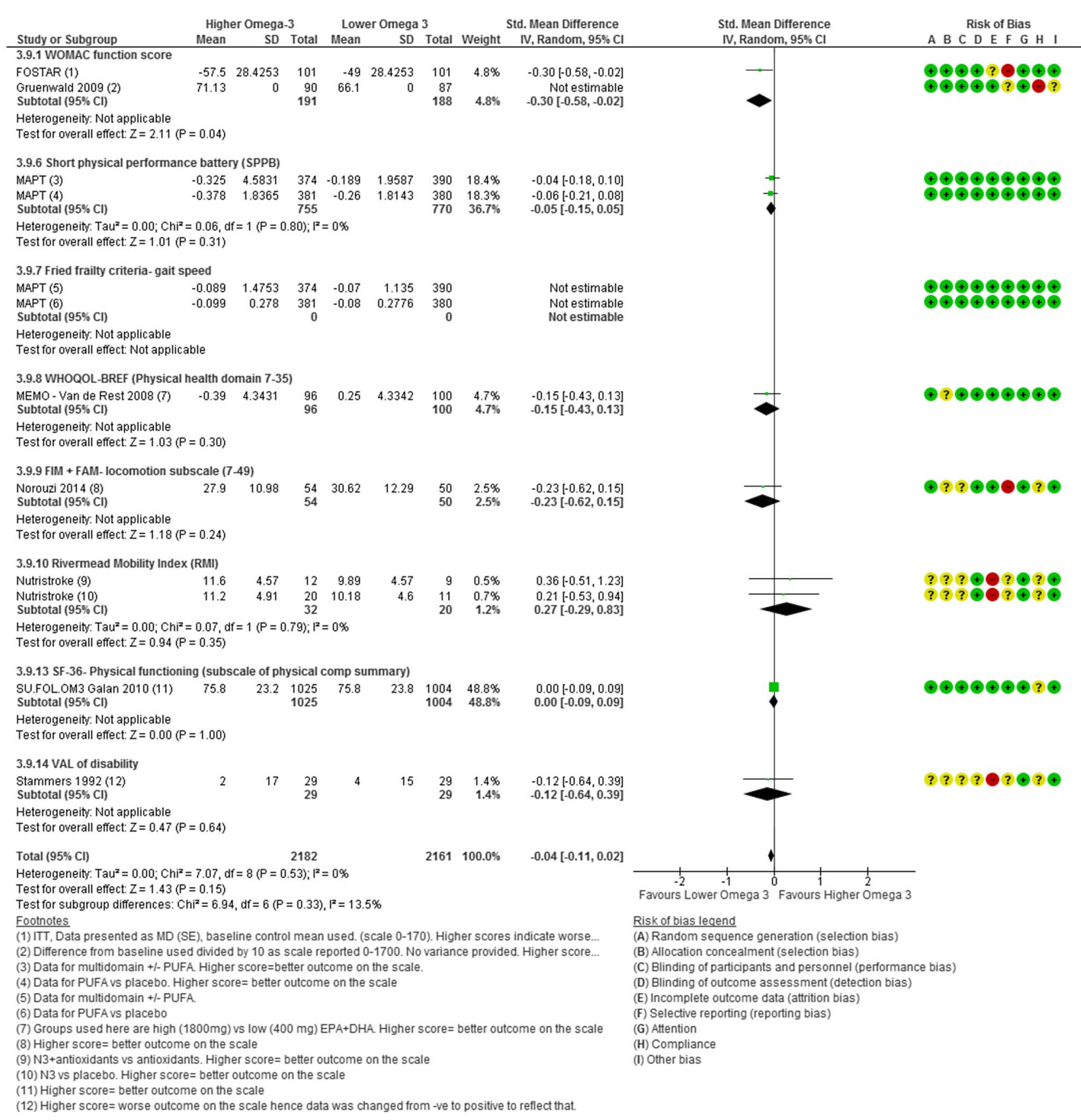

Fig. 5 Effect of high versus low omega-3 on various measures of functional status

assessed within the two RCTs included leg extensor power, walking speed and repeated chair rises, but no single measure was reported by both studies. These two studies suggested improvement in the measures used with high omega-3 PUFA and combining data from the two studies using SMD suggested a positive effect of high omega-3 PUFA on physical performance (SMD 0.47, 95\% CI 0.09, 0.85, 161 participants, $\left.\mathrm{I}^{2} 17 \%\right)$.
Bone formation markers were reported in three studies, none of which was at low summary risk of bias. One study author provided raw data that allowed inclusion in the metaanalysis [22]. Osteocalcin was the most frequently reported marker ( 3 studies, 4 comparisons, with heterogeneous data, $\left.\mathrm{I}^{2} 55 \%\right)$ suggesting a small increase with increased omega3. There was little or no effect on other markers (Additional Table 1). 
Bone resorption markers were reported in four studies [22, 42, 45, 46]; C-terminal telopeptide of type 1 collagen (CTX) was reported in two studies (3 comparisons) whilst N-telopeptide of type 1 collagen (NTX) and urinary total pyridinoline (PYD) in one study each. There was a suggestion of a decrease of CTX with increased omega-3, and an increase in urinary PYD, but these were non-statistically significant, and from small studies. Only the smallest trial was at low summary risk of bias and suggested no effect of omega-3 on CTX.

Various other markers of bone turnover were reported with parathyroid hormone being the most commonly reported (3 RCTs, 4 comparisons) with no significant effect of high omega-3 (Additional Table 1).

\section{Effects of Omega-6}

\section{Primary Outcomes}

No omega-6 studies reported on any primary outcome, so GRADE assessment was not carried out. Meta-analysis results are shown in Additional Table 2.

\section{Secondary Outcomes}

The only included omega-6 study [49] used GLA supplementation, randomised 84 participants and was not at low summary risk of bias. It provided data on two outcomes related to physical performance measures, arm and leg muscle strength, using scales of 0 to 2500 or 2000 , respectively, suggesting little or no effect. The paper is unclear about whether higher or lower scores indicated greater strength, though both measures suggested small statistically significant increases (Additional Table 2).

\section{Effects of Mixed Total PUFA}

GRADE assessment of quality of evidence is shown in Table 3, and meta-analysis results in Additional Table 3.

\section{Primary Outcomes}

No trials assessing effects on fracture incidence, total bone mass, BMC, sarcopenia, dynapenia or myopenia incidence or measures of functional status were identified.

\section{Bone Density}

Increasing total PUFA may have little or no effect on BMD (low-grade evidence, downgraded once each for risk of bias and indirectness). Three mixed PUFA studies reported BMD as an outcome (Fig. 6), but no two studies reported on the same site (total, femoral neck, lumbar or calcaneal reported).
Combining the three trials across sites (allowing one entry from the study with multiple measures) suggested little or no effect ( $<2 \%$ change from baseline). For individual sites, there was a suggestion that increasing total PUFA increased femoral neck BMD (MD $0.07 \mathrm{~g} / \mathrm{cm}^{2}, 95 \%$ CI 0.03 to $0.1,1$ RCT, 60 participants), but there were no suggested effects at any other site. No studies were at low summary risk of bias.

\section{Skeletal Muscle Mass}

Increasing total PUFA may have little or no effect on fat-free mass (downgraded once each for risk of bias and indirectness). Fat-free mass was reported in one study [50] with 214 participants, and change from baseline was $<2 \%$ (MD $-0.5 \mathrm{~kg}, 95 \% \mathrm{CI}-1.63$ to 0.63 ).

\section{Secondary Outcomes}

No included trial reporting secondary outcomes was at low summary risk of bias.

\section{Measures of Physical Performance/Strength}

Only one small UK study of healthy post-menopausal women [38] at moderate-to-high summary risk of bias reported a small non-significant decline in leg extensor power in the mixed PUFA intervention group compared to the control (MD $-8 \mathrm{~W}, 95 \% \mathrm{CI}-23.8-7.8,42$ participants).

\section{Bone Turnover Markers}

Bone formation markers were reported in three studies, none at low summary risk of bias, with little or no effect for all except a suggestion of a reduction in C1NP with higher PUFA (Additional Table 3). Osteocalcin was reported by all three studies suggesting little or no effect (MD $0.52 \mu \mathrm{g} / \mathrm{L}$, 95\%CI - 1.99-0.95, 195 participants, $\mathrm{I}^{2} 45 \%$ ). Serum bonespecific alkaline phosphatase was reported in all three studies, but one study reported it as a percentage (so could not be combined). There was no significant effect on either measure. Other markers reported by a single study included serum type 1 procollagen and procollagen.

Various markers of bone resorption were reported in three studies, but none were at low summary risk of bias. All suggested little or no effect except that there were small non-statistically significant falls of NTX/CR and CTX and urinary hydroxyproline with higher PUFA intake. The deoxypyridinoline/creatinine ratio was the only marker reported in two studies, other markers were reported by single studies. Parathyroid hormone was the most frequently reported bone turnover marker, reported by three studies. Pooled results suggested a small non-statistically significant increase in parathyroid hormone with increased PUFA. 


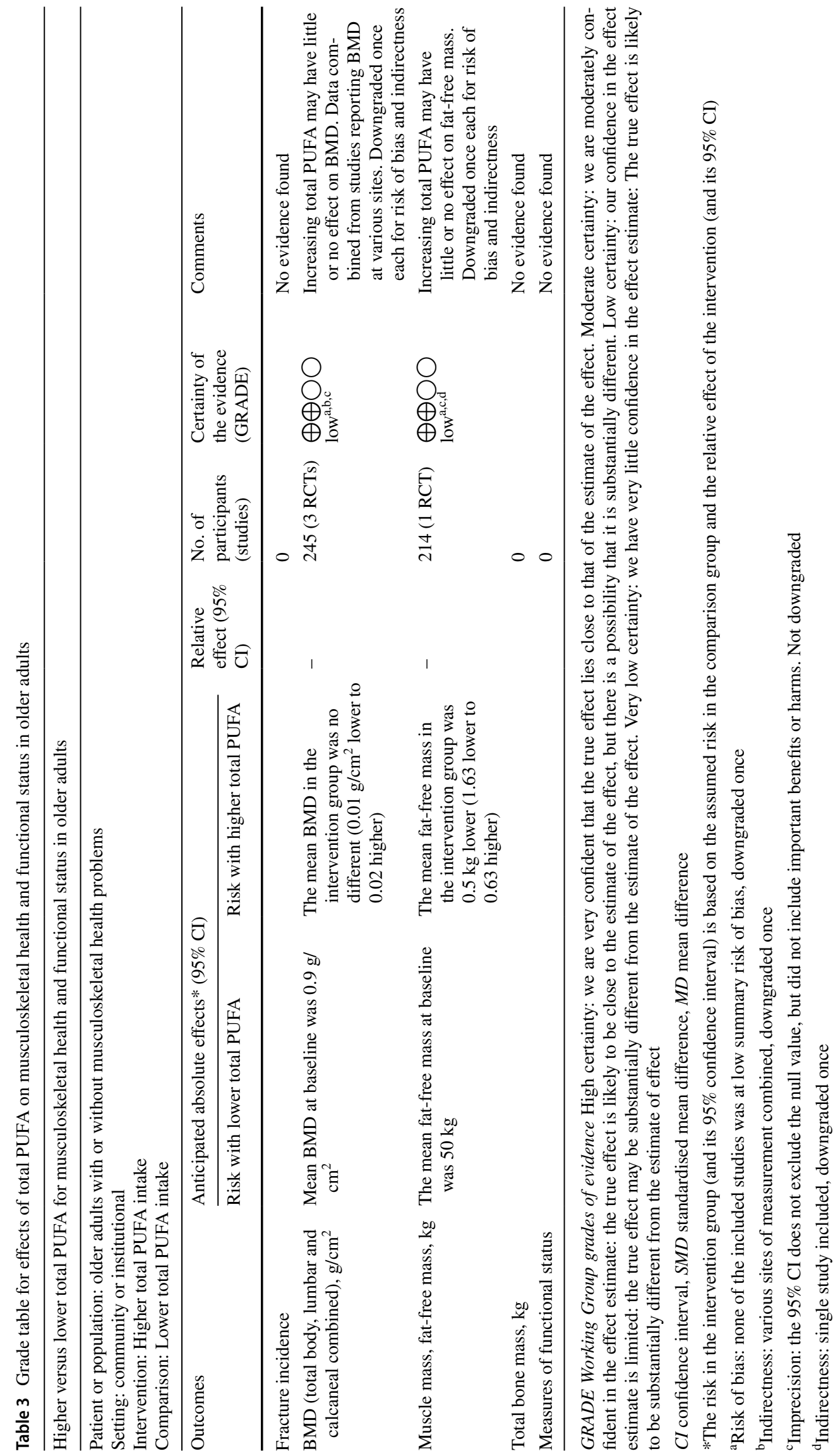




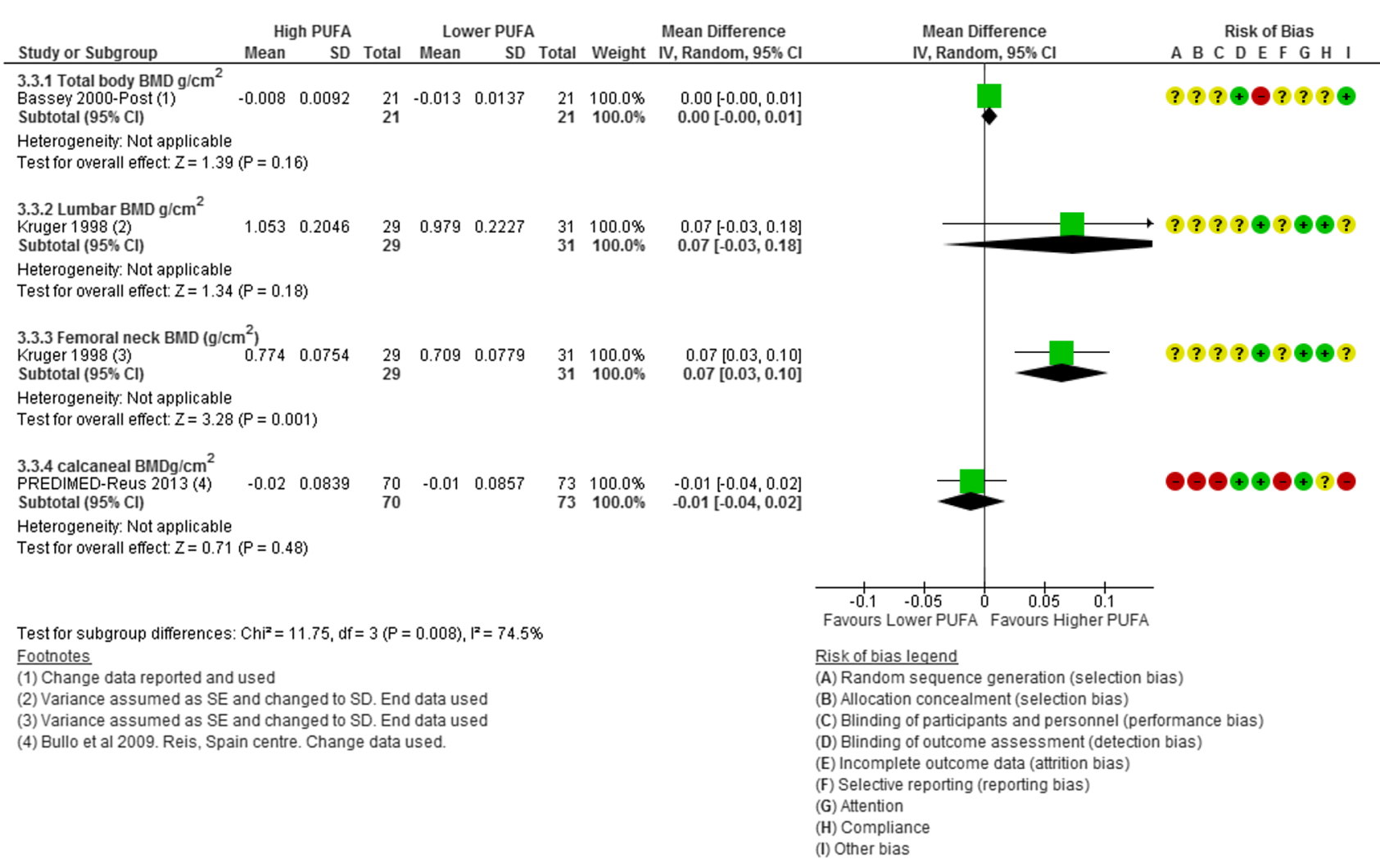

Fig. 6 Effect of high versus low mixed PUFA on BMD in different sites

\section{Discussion}

We have systematically reviewed the long-term effects of omega-3, omega-6 and total PUFA supplementation on risk factors for sarcopenia and fracture: functional status, bone density and skeletal muscle mass. We identified and included 28 RCTs (31 comparisons, 7288 participants), of which six were at low summary risk of bias. Twenty-three studies reported on the effects of increasing omega- 3 fatty acids, but effects were unclear (as the evidence was of very low quality) for fracture incidence, total proximal femur BMD and skeletal muscle mass. Low or very low-quality data suggested that increasing omega-3 may increase lumbar spine and femoral neck BMD but has little or no effect on total bone mass or measures of functional status. A few small trials suggested increases in physical performance and osteocalcin with increased omega-3 but data were of limited quality. The single eligible study on omega-6 did not report on any primary outcomes, and was not at low summary risk of bias. As far as it could be interpreted, this study suggested little or no effect on arm and leg muscle strength of omega-6. Three RCTs (4 comparisons) assessed effects of increasing mixed total PUFA and none were at low summary risk of bias. None reported on fracture incidence, total bone mass or measures of functional status. The existing data suggested that increasing total PUFA may have little or no effect on BMD (when effects at different sites were combined) or fatfree mass. Data on secondary outcomes were very limited but suggested reductions in leg extensor power, C1NP, NTX/ CR, CTX and urinary hydroxyproline, and a small increase in parathyroid hormone with more PUFA. There was considerable heterogeneity in populations studied and doses of fatty acids supplemented.

Observational data have suggested positive associations between intake of total PUFAs, total omega- 6 and total omega-3 and BMD [61, 62]. This systematic review suggests that increasing omega-3 has little or no effect on functional status, though it may increase BMD a little. There is some evidence from reviews of shorter duration trials that omega-3 improves skeletal muscle outcomes [21] and decreases osteocalcin [19]. However, if such effects are not maintained over the longer term their utility is very limited.

To our knowledge, this is the first systematic review to evaluate all PUFA interventions on both bone and skeletal muscle health as well as functional status, important risk factors for sarcopenia, osteoporosis and increased fragility fractures. This review included trials irrespective of whether their primary aim was to assess skeletal muscle or bone outcomes. This allowed us to evaluate effects of PUFA supplementation on various measures of sarcopenia. 
This was beneficial as sarcopenia has been poorly defined until recently [63, 64], and no studies assessed sarcopenia as a diagnosis. Our review excluded studies with multifactorial or multi-supplement interventions. Although this limited the evidence base used, it ensures that any effects seen are specifically related to PUFA supplementation. Our minimum intervention period was 24 weeks, as previous studies have suggested this length of time is the minimum required to allow equilibration of most body compartments with an altered fatty acid balance, and allow time for bone and muscle changes to become detectable $[65,66]$. Limitations included limited numbers of relevant trials, and limited numbers of trials at low summary risk of bias. Whilst we excluded studies in individuals with overt clinical conditions that may influence the metabolism and utilisation of unsaturated fatty acids, not all studies described the proportion of individuals with, for instance, type 2 diabetes. Whilst the presence of type 2 diabetes may influence the results of supplementation studies with PUFA, we were unable to quantify these effects in the data available. This, along with heterogeneous results, has led to the evidence produced being of low or very low quality so overall the evidence to address effects of omega-3, omega- 6 and total PUFA on functional, muscle and bone outcomes is limited. The inclusion of all PUFA nutritional interventions regardless of their nature (i.e. supplemental, food provision or dietary advice) could be viewed as a limitation restricting the comparability of the results, however, all interventions in the included studies aimed to increase one or more PUFA.

Despite the limitations of our study due to the lack of comprehensive evidence from trials with low risk of bias, thus limiting the conclusions from our study the importance of PUFA on musculoskeletal heath and outcomes deserves further research. The lack of studies relating greater intakes of PUFA to risk factors for sarcopenia is due to the relatively recent recognition and evolving definitions for this condition [64] as well as to the combination of exercise and dietary interventions which means it is not possible to isolate the effects of PUFA intake alone.

Recommendations for future research include largerscale studies in populations likely to have low intakes as well as circulating concentrations of EPA and DHA. ALA interventions are also unlikely to be effective in those already consuming high concentrations of EPA and DHA due to their competition with ALA as substrates for the desaturase, elongase pathway responsible for conversion from ALA to EPA and then DHA [67]. So these studies should be limited to populations consuming small quantities of EPA and DHA. Future studies determining the effects of omega-3 PUFA on BMD would require a duration of 12 months or longer to enable detection of measurable changes and the intervention to be of sufficient scale to detect the effects these interventions. We also recommend taking baseline measurements of intake and circulating concentrations of PUFA. Reporting from trials should also include the proportion of individuals with metabolic conditions such as type 2 diabetes that may impact on the utilisation and metabolism of PUFA.

In conclusion, we found low-quality evidence that omega-3 may increase BMD by a small amount, but there were no other clear effects of omega-3 or total PUFA on skeletal muscle, bone or functional outcomes. Evidence of the effects of omega- 6 supplementation on bone or skeletal muscle outcomes was insufficient to warrant any conclusions. Further trials assessing effects of omega-3, omega-6 and mixed PUFA on musculoskeletal outcomes are warranted, but only if the trials are methodologically strong (at low summary risk of bias) and appropriately powered.

Acknowledgements This review is one of a set of reviews conducted by the PUFAH group. The Polyunsaturated Fats and Health (PUFAH) group includes Asmaa Abdelhamid, Norwich Medical School, University of East Anglia; Sarah Ajabnoor, Norwich Medical School, University of East Anglia; Faye Alabdulghafoor, Norwich Medical School, University of East Anglia; Lena Alkhudairy, Warwick Medical School, University of Warwick; Priti Biswas, Health Sciences, University of East Anglia; Julii Brainard, Norwich Medical School, University of East Anglia; Charlene Bridges, Cochrane Heart Group, University College London; Tracey J Brown, Norwich Medical School, University of East Anglia; Katherine Deane, Health Sciences, University of East Anglia; Daisy Donaldson, Norwich Medical School, University of East Anglia; Sarah Hanson, Health Sciences, University of East Anglia; Lee Hooper, Norwich Medical School, University of East Anglia; Oluseyi Florence Jimoh, Norwich Medical School, University of East Anglia; Nicole Martin, Cochrane Heart Group, University College London; Alex O'Brien, Norwich Medical School, University of East Anglia; Karen Rees, Lena Alkhudairy, Warwick Medical School, University of Warwick; Fujian Song, Norwich Medical School, University of East Anglia; Gabrielle Thorpe, Health Sciences, University of East Anglia; Xia Wang , Norwich Medical School, University of East Anglia and Lauren Winstanley, Norwich Medical School, University of East Anglia. The review authors thank all of the authors of primary studies who kindly provided us with the best set of data available, including $\mathrm{P}$ Salari Sharif, Tehran University of Medical Sciences [22].

Author Contributions AW, AA and LH conceived this review; AA wrote the first draft of the protocol and submitted the protocol draft to PROSPERO; LH drafted the searches which were developed, refined, run and de-duplicated by the Cochrane Heart Group. AA and LH assessed full text papers for inclusion; LH searched trials registers and assessed entries for inclusion; AA and RS carried out data extraction and assessed risk of bias. LH and AA carried out data checks; AA and LH entered data into RevMan, ran meta-analyses and carried out sensitivity analyses. AA wrote the first draft of the review. AA and LH carried out GRADE assessment and interpretation. All authors contributed to the manuscript and agreed the final version.

Funding The World Health Organization (WHO) Nutrition Guidance Expert Advisory Group (NUGAG) Subgroup on Diet and Health funded the creation of the main database. No additional funding was used in completing this systematic review. 


\section{Compliance with Ethical Standards}

Conflict of interest All authors have completed the Osteoporosis International's authorship and disclosure form and declare: some authors had financial support via the University of East Anglia from the World Health Organization for the submitted work, and LH and AA were funded to attend WHO meetings to present the results of other reviews in this series; no financial relationships with any organisations that might have an interest in the submitted work in the previous three years; no other relationships or activities that could appear to have influenced the submitted work.

Ethical Approval No ethical approval was required.

Human and Animal Rights and Informed Consent This article does not contain any studies with human participants or animals performed by any of the authors. Therefore, no consent form was needed.

Open Access This article is distributed under the terms of the Creative Commons Attribution 4.0 International License (http://creativeco mmons.org/licenses/by/4.0/), which permits unrestricted use, distribution, and reproduction in any medium, provided you give appropriate credit to the original author(s) and the source, provide a link to the Creative Commons license, and indicate if changes were made.

\section{References}

1. Gillette-Guyonnet S, Nourhashemi F, Lauque S, Grandjean H, Vellas B (2000) Body composition and osteoporosis in elderly women. Gerontology 46:189-193

2. Iannuzzi-Sucich M, Prestwood KM, Kenny AM (2002) Prevalence of sarcopenia and predictors of skeletal muscle mass in healthy, older men and women. J Gerontol Ser A 57:M772-777

3. Lauretani F, Russo CR, Bandinelli S, Bartali B, Cavazzini C, Iorio AD, Corsi AM, Rantanen T, Guralnik JM, Ferrucci L (2003) Age-associated changes in skeletal muscles and their effect on mobility: an operational diagnosis of sarcopenia. J Appl Physiol 95:1851-1860

4. Fried LP, Guralnik JM (1997) Disability in older adults: evidence regarding significance, etiology, and risk. J Am Geriatr Soc 45:92-100

5. Hunter DJ, Sambrook PN (2000) Bone loss: epidemiology of bone loss. Arthritis Res Ther 2:441-445

6. Metter EJ, Conwit R, Tobin J, Fozard JL (1997) Age-associated loss of power and strength in the upper extremities in women and men. J Gerontol Ser A 52:B267-276

7. Hernlund E, Svedbom A, Ivergard M, Compston J, Cooper C, Stenmark J, McCloskey EV, Jonsson B, Kanis JA (2013) Osteoporosis in the European Union: medical management, epidemiology and economic burden. A report prepared in collaboration with the International Osteoporosis Foundation (IOF) and the European Federation of Pharmaceutical Industry Associations (EFPIA). Arch Osteoporos 8:136

8. Sousa AS, Guerra RS, Fonseca I, Pichel F, Ferreira S, Amaral TF (2016) Financial impact of sarcopenia on hospitalization costs. Eur J Clin Nutr 70:1046-1051

9. Ethgen O, Beaudart C, Buckinx F, Bruyere O, Reginster JY (2016) The future prevalence of sarcopenia in Europe: a claim for public health action. Calcif Tissue Int 100:229-234

10. United Nations Department of Economic Social Affairs (2017) World population prospects: The 2017 revision, key findings and advance tables. Working Paper No. ESA/P/WP/248, Accessed 2017
11. Melzer K (2011) Carbohydrate and fat utilization during rest and physical activity. e-SPEN 6:45-52

12. Lipina C, Hundal HS (2017) Lipid modulation of skeletal muscle mass and function. J Cachexia Sarcopenia Muscle 8:190-201

13. Mangano KM, Sahni S, Kerstetter JE, Kenny AM, Hannan MT (2013) Polyunsaturated fatty acids and their relation with bone and muscle health in adults. Curr Osteoporos Rep 11:203-212

14. Tachtsis B, Camera D, Lacham-Kaplan O (2018) Potential roles of n-3 PUFAs during skeletal muscle growth and regeneration. Nutrients 10:309

15. Maurin AC, Chavassieux PM, Meunier PJ (2005) Expression of PPARgamma and beta/delta in human primary osteoblastic cells: influence of polyunsaturated fatty acids. Calcif Tissue Int 76:385-392

16. Haag M, Magada ON, Claassen N, Bohmer LH, Kruger MC (2003) Omega-3 fatty acids modulate ATPases involved in duodenal $\mathrm{Ca}$ absorption. Prostaglandins Leukot Essent Fatty Acids 68:423-429

17. Young LR, Kurzer MS, Thomas W, Redmon JB, Raatz SK (2013) Low fat diet with omega-3 fatty acids increases plasma insulinlike growth factor concentration in healthy postmenopausal women(1). Nutr Res 33:565-571

18. Paunescu A-C, Dewailly É, Dodin S, Nieboer E, Ayotte P (2013) Dioxin-like compounds and bone quality in Cree women of Eastern James Bay (Canada): a cross-sectional study. Environmental Health 12:54

19. Shen D, Zhang X, Li Z, Bai H, Chen L (2017) Effects of omega-3 fatty acids on bone turnover markers in postmenopausal women: systematic review and meta-analysis. Climacteric: the journal of the International Menopause Society 20:522-527

20. Smith GI (2016) The Effects of Dietary Omega-3 s on Muscle Composition and Quality in Older Adults. Current Nutrition Reports 5:99-105

21. Buoite Stella A, Gortan Cappellari G, Barazzoni R, Zanetti M (2018) Update on the Impact of Omega 3 Fatty Acids on Inflammation, Insulin Resistance and Sarcopenia: A Review. International journal of molecular sciences 19

22. Salari Sharif P, Asalforoush M, Ameri F, Larijani B, Abdollahi M (2010) The effect of $n-3$ fatty acids on bone biomarkers in Iranian postmenopausal osteoporotic women: a randomized clinical trial. Age (Dordr) 32:179-186

23. Orchard TS, Pan X, Cheek F, Ing SW, Jackson RD (2012) A systematic review of omega-3 fatty acids and osteoporosis. Br J Nutr 107:S253-S260

24. Albertazzi P, Coupland K (2002) Polyunsaturated fatty acids. Is there a role in postmenopausal osteoporosis prevention? Maturitas 42:13-22

25. Moher D, Liberati A, Tetzlaff J, Altman DG (2009) Preferred reporting items for systematic reviews and meta-analyses: the PRISMA statement. PLoS Med 6:e1000097

26. Higgins JP, Green S Cochrane Handbook for Systematic Reviews of Interventions Version 5.1.0 (updated March 2011). The Cochrane Collaboration, 2011. www.handbook.cochrane.org

27. Abdelhamid AS, Brown TJ, Brainard JS, Biswas P, Thorpe GC, Moore HJ, Deane KH, AlAbdulghafoor FK, Summerbell CD, Worthington HV, Song F, Hooper L (2018) Omega-3 fatty acids for the primary and secondary prevention of cardiovascular disease. Cochrane Datab Syst Rev. https://doi.org/10.1002/14651858. CD003177.pub3

28. Abdelhamid AS, Martin N, Bridges C, Brainard JS, Wang X, Brown TJ, Hanson S, Jimoh OF, Ajabnoor SM, Deane KH, Song F, Hooper L (2018) Polyunsaturated fatty acids for the primary and secondary prevention of cardiovascular disease. Cochrane Datab Syst Rev. https://doi.org/10.1002/14651858.CD003177. pub3 
29. Brown TJ, Brainard JS, Song F, Wang X, Abdelhamid A, Hooper L, PUFAH Group (2019) Omega-3, omega-6 and total dietary polyunsaturated fat for prevention and treatment of type 2 diabetes mellitus: systematic review and meta-analysis of randomised controlled trials. Br Med J

30. Deane KHO, Jimoh OF, Biswas P, O'Brien AT, Hanson S, Abdelhamid A, Fox C, Hooper L (2019) Omega-3, omega-6 and total polyunsaturated fat for prevention of depression and anxiety symptoms: a systematic review and meta-analysis of randomised trials. Submitted

31. Hanson S, Thorpe G, Winstanley L, Abdelhamid A, Hooper L (2017) Effects of supplementary dietary polyunsaturated fat on cancer incidence. PROSPERO:CRD42017056109

32. Hooper L, Al-Khudairy L, Abdelhamid AS, Rees K, Brainard JS, Brown TJ, Ajabnoor SM, O'Brien AT, Winstanley LE, Donaldson DH, Song F, Deane KH (2018) Omega-6 fats for the primary and secondary prevention of cardiovascular disease. Cochrane Datab Syst Rev. https://doi.org/10.1002/14651858. CD011094.pub3

33. Brainard J, Jimoh OF, Deane K, Biswas P, Donaldson D, Maas K, Abdelhamid A, Hooper L (2019) Omega-3, omega-6 and total polyunsaturated fat for cognition and dementia: systematic review and meta-analysis of RCTs

34. Thorpe G, Ajabnoor S, Ahmed Z, Abdelhamid A, Hooper L (2017) Dietary polyunsaturated fat for prevention and treatment of inflammatory bowel disease. PROSPERO:CRD42017068704

35. Hooper L, Abdelhamid A, Brainard J, Deane KHO, Song F, Group P (2019) Creation of a database to assess effects of omega3 , omega- 6 and total polyunsaturated fats on health: methodology for a set of systematic reviews. BMJ Open 9:e029554

36. Higgins JPT, Altman DG, Gøtzsche PC, Jüni P, Moher D, Oxman AD, Savović J, Schulz KF, Weeks L, Sterne JAC (2011) The Cochrane Collaboration's tool for assessing risk of bias in randomised trials. Br Med J 343:d5928

37. Welch AA, Skinner J, Hickson M (2017) Dietary magnesium may be protective for aging of bone and skeletal muscle in middle and younger older age men and women: cross-sectional findings from the UK Biobank cohort. Nutrients 9:E1189

38. Bassey EJ, Littlewood JJ, Rothwell MC, Pye DW (2000) Lack of effect of supplementation with essential fatty acids on bone mineral density in healthy pre- and postmenopausal women: two randomized controlled trials of Efacal v. calcium alone. Br J Nutr 83:629-635

39. Baxheinrich A, Stratmann B, Lee-Barkey Y, Tschoepe D, Wahrburg U (2012) Effects of a rapeseed oil-enriched hypoenergetic diet with a high content of alpha-linolenic acid on body weight and cardiovascular risk profile in patients with the metabolic syndrome. Br J Nutr 108:682-691

40. Dodin S, Lemay A, Jacques H, Legare F, Forest JC, Masse B (2005) The effects of flaxseed dietary supplement on lipid profile, bone mineral density, and symptoms in menopausal women: a randomized, double-blind, wheat germ placebo-controlled clinical trial. J Clin Endocrinol Metab 90:1390-1397

41. Dong H, Hutchins-Wiese H, Kleppinger A, Annis K, Liva E, Lammi-Keefe C, Durham H, Feinn R, Kenny AM (2014) Effects of omega-3 polyunsaturated fatty acid supplementation on bone turnover in older women. Int J Vitam Nutr Res 84:124-132

42. Hutchins-Wiese HL, Kleppinger A, Annis K, Liva E, LammiKeefe CJ, Durham HA et al (2013) The impact of supplemental n-3 long chain polyunsaturated fatty acids and dietary antioxidants on physical performance in postmenopausal women. J Nutr Health Aging 17:76-80

43. Kruger MC, Coetzer H, de Winter R, Gericke G, van Papendorp DH (1998) Calcium, gamma-linolenic acid and eicosapentaenoic acid supplementation in senile osteoporosis. Aging (Milan, Italy) $10: 385-394$
44. Tardivo AP, Nahas-Neto J, Orsatti CL, Dias FB, Poloni PF, Schmitt EB, Nahas EA (2015) Effects of omega-3 on metabolic markers in postmenopausal women with metabolic syndrome. Climacteric 18:290-298

45. Tartibian B, Hajizadeh Maleki B, Kanaley J, Sadeghi K (2011) Long-term aerobic exercise and omega-3 supplementation modulate osteoporosis through inflammatory mechanisms in postmenopausal women: a randomized, repeated measures study. Nutr Metab 8:71-71

46. Vanlint SJ, Ried K (2012) Efficacy and tolerability of calcium, vitamin D and a plant-based omega-3 oil for osteopenia: a pilot RCT. Maturitas 71:44-48

47. Bullo M, Amigo-Correig P, Marquez-Sandoval F, Babio N, Martinez-Gonzalez MA, Estruch R, Basora J, Sola R, SalasSalvado J (2009) Mediterranean diet and high dietary acid load associated with mixed nuts: effect on bone metabolism in elderly subjects. J Am Geriatr Soc 57:1789-1798

48. Fernandez-Real JM, Bullo M, Moreno-Navarrete JM, Ricart W, Ros E, Estruch R et al (2012) A Mediterranean diet enriched with olive oil is associated with higher serum total osteocalcin levels in elderly men at high cardiovascular risk. J Clin Endocrinol Metab 97:3792-3798

49. Keen H, Payan J, Allawi J, Walker J, Jamal GA, Weir AI et al (1993) Treatment of diabetic neuropathy with gamma-linolenic acid. The gamma-Linolenic Acid Multicenter Trial Group. Diabetes Care 16:8-15

50. Alvarez-Perez J, Sanchez-Villegas A, Diaz-Benitez EM, RuanoRodriguez C, Corella D, Martinez-Gonzalez MA, Estruch R, Salas-Salvado J, Serra-Majem L, Investigators PS (2016) Influence of a mediterranean dietary pattern on body fat distribution: results of the PREDIMED-canarias intervention randomized trial. J Am Coll Nutr 35:1-13

51. Stammers T, Sibbald B, Freeling P (1992) Efficacy of cod liver oil as an adjunct to non-steroidal anti-inflammatory drug treatment in the management of osteoarthritis in general practice. Ann Rheum Dis 51:128-129

52. Danthiir V, Hosking D, Burns NR, Wilson C, Nettelbeck T, Calvaresi E et al (2014) Cognitive performance in older adults is inversely associated with fish consumption but not erythrocyte membrane n-3 fatty acids. J Nutr 144:311-320

53. Sinn N, Milte CM, Street SJ, Buckley JD, Coates AM, Petkov J, Howe PR (2012) Effects of n-3 fatty acids, EPA v. DHA, on depressive symptoms, quality of life, memory and executive function in older adults with mild cognitive impairment: a 6-month randomised controlled trial. Br J Nutr 107:1682-1693

54. Faxén Irving G, Freund-Levi Y, Eriksdotter-Jönhagen M, Basun H, Brismar K, Hjorth E, Palmblad J, Vessby B, Vedin I, Wahlund L, Cederholm T (2009) N-3 fatty acid supplementation effects on weight and appetite in patients with Alzheimer's disease: the OmegAD Study. J Am Geriatr Soc 57:11-17

55. Andrieu S, Guyonnet S, Coley N, Cantet C, Bonnefoy M, Bordes $S$ et al (2017) Effect of long-term omega 3 polyunsaturated fatty acid supplementation with or without multidomain intervention on cognitive function in elderly adults with memory complaints (MAPT): a randomised, placebo-controlled trial. Lancet Neurol 16:377-389

56. Galan P, Briancon S, Blacher J, Czernichow S, Hercberg S (2008) The SU.FOL.OM3 Study: a secondary prevention trial testing the impact of supplementation with folate and B-vitamins and/or Omega-3 PUFA on fatal and non fatal cardiovascular events, design, methods and participants characteristics. Trials 9:35-35

57. Galan P, Kesse-Guyot E, Czernichow S, Briancon S, Blacher J, Hercberg S, Group SFOC (2010) Effects of B vitamins and omega 3 fatty acids on cardiovascular diseases: a randomised placebo controlled trial. BMJ 341:c6273 
58. Gruenwald J, Petzold E, Busch R, Petzold HP, Graubaum HJ (2009) Effect of glucosamine sulfate with or without omega-3 fatty acids in patients with osteoarthritis. Adv Ther 26:858-871

59. Hill CL, March LM, Aitken D, Lester SE, Battersby R, Hynes K et al (2016) Fish oil in knee osteoarthritis: a randomised clinical trial of low dose versus high dose. Ann Rheum Dis 75:23-29

60. Dasarathy S, Dasarathy J, Khiyami A, Yerian L, Hawkins C, Sargent R, McCullough A (2015) Double blind randomized placebo controlled clinical trial of omega 3 fatty acids for the treatment of diabetic patients with nonalcoholic steatohepatitis. J Clin Gastroenterol 49:137-144

61. Järvinen R, Tuppurainen M, Erkkilä AT, Penttinen P, Kärkkäinen M, Salovaara K, Jurvelin JS, Kröger H (2011) Associations of dietary polyunsaturated fatty acids with bone mineral density in elderly women. Eur J Clin Nutr 66:496

62. Longo AB, Ward WE (2016) PUFAs, Bone mineral density, and fragility fracture: findings from human studies. Adv Nutr 7:299-312

63. Cruz-Jentoft AJ, Baeyens JP, Bauer JM, Boirie Y, Cederholm T, Landi F, Martin FC, Michel JP, Rolland Y, Schneider SM, Topinková E, Vandewoude M, Zamboni M, European Working Group on Sarcopenia in Older People (2010) Sarcopenia: European consensus on definition and diagnosis: Report of the European Working Group on Sarcopenia in Older People. Age Ageing 39:412-423

64. Cruz-Jentoft AJ, Bahat $\mathrm{G}$, Bauer J, Boirie $\mathrm{Y}$, Bruyère $\mathrm{O}$, Cederholm T, Cooper C, Landi F, Rolland Y, Sayer AA, Schneider SM, Sieber CC, Topinkova E, Vandewoude M, Visser M, Zamboni M, Writing Group for the European Working Group on Sarcopenia in Older P, the Extended Group for E (2019) Sarcopenia: revised European consensus on definition and diagnosis. Age Ageing 48:16-31

65. Browning LM, Walker CG, Mander AP, West AL, Madden J, Gambell JM et al (2012) Incorporation of eicosapentaenoic and docosahexaenoic acids into lipid pools when given as supplements providing doses equivalent to typical intakes of oily fish. Am J Clin Nutr 96:748-758

66. Walker CG, Browning LM, Stecher L, West AL, Madden J, Jebb SA, Calder PC (2015) Fatty acid profile of plasma NEFA does not reflect adipose tissue fatty acid profile. Br J Nutr 114:756-762

67. Welch AA, Shakya-Shrestha S, Lentjes MA, Wareham NJ, Khaw KT (2010) Dietary intake and status of n-3 polyunsaturated fatty acids in a population of fish-eating and non-fish-eating meat-eaters, vegetarians, and vegans and the product-precursor ratio [corrected] of alpha-linolenic acid to long-chain $n-3$ polyunsaturated fatty acids: results from the EPIC-Norfolk cohort. Am J Clin Nutr 92:1040-1051

68. Clark LF, Thivierge MC, Kidd CA, McGeoch SC, Abraham P, Pearson DW, Horgan GW, Holtrop G, Thies F, Lobley GE (2016) Fish oil supplemented for 9 months does not improve glycaemic control or insulin sensitivity in subjects with impaired glucose regulation: a parallel randomised controlled trial. Br J Nutr 115:75-86

69. Danthiir V, Burns NR, Nettelbeck T, Wilson C, Wittert G (2011) The older people, omega-3, and cognitive health $(\mathrm{EPOCH})$ trial design and methodology: a randomised, double-blind, controlled trial investigating the effect of long-chain omega- 3 fatty acids on cognitive ageing and wellbeing in cognitively healthy older adults. Nutr J 10:117-117

70. Chen JS, Hill CL, Lester S, Ruediger CD, Battersby R, Jones G, Cleland LG, March LM (2016) Supplementation with omega-3 fish oil has no effect on bone mineral density in adults with knee osteoarthritis: a 2-year randomized controlled trial. Osteoporos Int 27:1897-1905

71. Van De Rest O, Geleijnse JM, Kok FJ, van Staveren WA, Dullemeijer C, Olderikkert MG, Beekman AT, De Groot CP (2008) Effect of fish oil on cognitive performance in older subjects: a randomized, controlled trial. Neurology 71:430-438

72. Norouzi Javidan A, Sabour H, Latifi S, Abrishamkar M, Soltani Z, Shidfar F, Emami Razavi H (2014) Does consumption of polyunsaturated fatty acids influence on neurorehabilitation in traumatic spinal cord-injured individuals? A double-blinded clinical trial. Spinal Cord 52:378-382

73. Garbagnati F, Cairella G, De Martino A, Multari M, Scognamiglio $\mathrm{U}$, Venturiero V et al (2009) Is antioxidant and n-3 supplementation able to improve functional status in poststroke patients? Results from the Nutristroke Trial. Cerebrovasc Dis 27:375-383

74. Freund Levi Y, Eriksdotter-Jönhagen M, Cederholm T, Basun H, Faxén Irving G, Garlind A, VedinI Vessby B, Wahlund LO, Palmblad J (2006) Omega-3 fatty acid treatment of 174 patients with mild to moderate Alzheimer's disease (OmegAD): a randomized double-blind trial. Arch Neurol 63:1402-1408

75. Smith GI, Julliand S, Reeds DN, Sinacore DR, Klein S, Mittendorfer B (2015) Fish oil-derived n-3 PUFA therapy increases muscle mass and function in healthy older adults. Am J Clin Nutr 102:115-122

76. Wang F, Wang Y, Zhu Y, Liu X, Xia H, Yang X (2016) Treatment for 6 months with fish oil-derived n-3 polyunsaturated fatty acids has neutral effects on glycemic control but improves dyslipidemia in type 2 diabetic patients with abdominal obesity: a randomized, double-blind, placebo-controlled trial. Eur J Nutr 1:1. https://doi. org/10.1007/s00394-016-1352-4

77. Scorletti E, Bhatia L, McCormick KG, Clough GF, Nash K, Hodson L et al (2014) Effects of purified eicosapentaenoic and docosahexaenoic acids in nonalcoholic fatty liver disease: results from the WELCOME study. Hepatology 60:1211-1221

78. Estruch R, Ros E, Salas-Salvadó J, Covas M, Corella D, Arós F et al (2018) Retraction and republication: primary prevention of cardiovascular disease with a Mediterranean diet. N Engl J Med 2013 368:1279-1290

Publisher's Note Springer Nature remains neutral with regard to jurisdictional claims in published maps and institutional affiliations.

\title{
Affiliations
}

\section{Asmaa Abdelhamid ${ }^{1} \cdot$ Lee Hooper ${ }^{1} \cdot$ Ruksana Sivakaran $^{1} \cdot$ Richard P. G. Hayhoe $^{1} \cdot$ Ailsa Welch $^{1}$ on behalf of the PUFAH Group}

\author{
Ruksana Sivakaran \\ r.sivakaran@uea.ac.uk \\ Richard P. G. Hayhoe \\ r.hayhoe@uea.ac.uk \\ Ailsa Welch \\ a.welch@uea.ac.uk
}

1 Norwich Medical School, University of East Anglia, 